STRUCTURAL BIOLOGY COMMUNICATIONS

ISSN 2053-230X

\section{The crystal structures of the enzyme hydroxymethylbilane synthase, also known as porphobilinogen deaminase}

\author{
John R. Helliwell*
}

Department of Chemistry, University of Manchester, Manchester M13 9PL, United Kingdom. *Correspondence e-mail: john.helliwell@manchester.ac.uk

Received 10 August 2021

Accepted 16 September 2021

Edited by M. J. van Raaij, Centro Nacional de Biotecnología - CSIC, Spain

This article is dedicated to the PDB on the occasion of the 50th anniversary of its commencement and, as just one example, to mark its careful curation of the hydroxymethylbilane synthase crystal structures cited in this article.

Keywords: hydroxymethylbilane synthase; porphobilinogen deaminase; enzyme-substrate intermediates; reaction mechanisms; structure and function.

Supporting information: this article has supporting information at journals.iucr.org/f

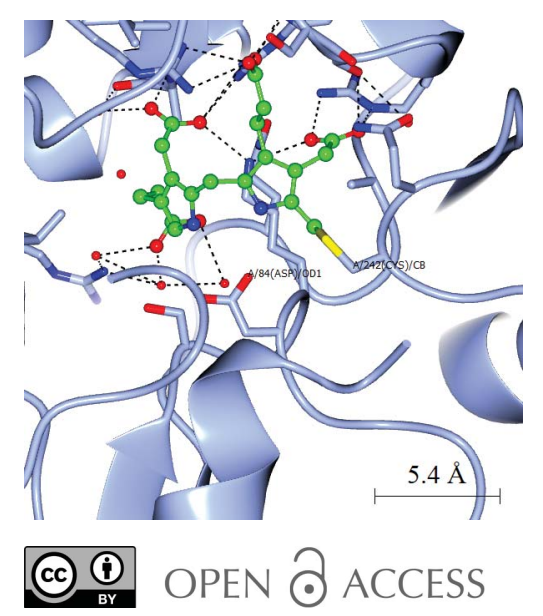

The enzyme hydroxymethylbilane synthase (HMBS; EC 4.3.1.8), also known as porphobilinogen deaminase, catalyses the stepwise addition of four molecules of porphobilinogen to form the linear tetrapyrrole 1-hydroxymethylbilane. Thirty years of crystal structures are surveyed in this topical review. These crystal structures aim at the elucidation of the structural basis of the complex reaction mechanism involving the formation of tetrapyrrole from individual porphobilinogen units. The consistency between the various structures is assessed. This includes an evaluation of the precision of each molecular model and what was not modelled. A survey is also made of the crystallization conditions used in the context of the operational $\mathrm{pH}$ of the enzyme. The combination of 3D structural techniques, seeking accuracy, has also been a feature of this research effort. Thus, SAXS, NMR and computational molecular dynamics have also been applied. The general framework is also a considerable chemistry research effort to understand the function of the enzyme and its medical pathologies in acute intermittent porphyria (AIP). Mutational studies and their impact on the catalytic reaction provide insight into the basis of AIP and are also invaluable for guiding the understanding of the crystal structure results. Future directions for research on HMBS are described, including the need to determine the protonation states of key amino-acid residues identified as being catalytically important. The question remains - what is the molecular engine for this complex reaction? Thermal fluctuations are the only suggestion thus far.

\section{Introduction}

The course of the reaction catalysed by hydroxymethylbilane synthase (HMBS) is depicted in Fig. 1 as well as its overall position in the pathway to uroporphyrinogen III (Hädener $e t$ al., 1999). Note the ammonia molecules released in each step. The crystal structure with PDB code 1ah5 (Hädener et al., 1999) is depicted in Fig. 2.

\section{Commentary on the role of the PDB data files in structural and functional studies of HMBS}

The details of each deposition are provided in Table 1. A synopsis of the role of each crystal structure is now provided. The HMBS structures studied are from multiple organisms; these are listed in Table 1. It is made clear to which organism the amino-acid numbering of the highlighted residues below refers.

$P D B$ entry 1pda. This is the opening, pioneering, crystal structure. It was of the inactive form of the enzyme. Louie $e t$ al. (1992) suggested that the enzyme mechanism has two options: the sliding of the catalytic residue Asp84 (Escherichia 
coli numbering) or the movement of the cofactor past Asp84, in which the relative motion of the individual domains is also likely to be involved.

$P D B$ entry 1ah5. This is the active form of the enzyme and was solved by selenomethionine MAD phasing using Station 9.5 at the SRS in the UK (Hädener et al., 1993, 1999). This study also gave early experience of the ESRF BM14 beamline and the ESRF image-intensifier detector (Cassetta et al., 1999). In Hädener et al. (1999), the SRS and ESRF MAD data and results are also compared.

$P D B$ entry 1ypn. This was the first experimental demonstration of the active-site location. It corresponds to the $2 \mathrm{~h}$ time point after reaction initiation by diffusion of porphobilinogen (PBG) into the crystal using a flow cell monitored by time-resolved diffraction on ID09 at ESRF. The experiment was based on the K59Q HMBS mutant, experiments using which had shown a buildup of the enzyme-substrate intermediate $\mathrm{ES}_{2}$ (Niemann et al., 1994). Extended electron density was established after $2 \mathrm{~h}$ in a geometric series of measured time-point data sets from $1 \mathrm{~min}$ to $12 \mathrm{~h}$. PBG fits the $2 \mathrm{~h}$ extended electron density $\left(2 F_{\mathrm{o}}-F_{\mathrm{c}}\right)$; this is likely to be the first reaction site $\left(\mathrm{ES}_{1}\right)$ as it is proximal to Asp84. The other end of the elongated density extended towards the side chain of Arg149, which is also an important residue. The maximum peak height was around $3.8 \sigma$ and the length of the peak was around $8 \AA$. The enzyme-substrate intermediate complexes require a covalent bond between the $\mathrm{C} 2$ ring of the cofactor and ring $\mathrm{A}$ of the polypyrrolic chain. However, the $2 \mathrm{~h}$ elongated density peak is not connected to the $\mathrm{C} 2$ ring of the cofactor; this point is discussed further below.
$P D B$ entry 2ypn. This was the time-zero data set that accompanied PDB entry 1ypn from the experiment on ID09 at ESRF.

$P D B$ entry 1gtk. This crystal structure determination was from diffraction data that were measured at $100 \mathrm{~K}$ and was compared with PDB entry 1ah5, the room-temperature crystal structure. However, the mobile loop consisting of residues 45-57, which sits close to the active site of HMBS, was not stabilized in PDB entry 1gtk. This loop was defined structurally in PDB entry 4htg, the Arabidopsis enzyme (Roberts et al., 2013), as well as being offered as a predicted structure by AlphaFold DB (Jumper et al., 2021).

$P D B$ entries 3ecr, 3eq1, 4htg, $4 \mathrm{mlq}$ and $4 \mathrm{mlv}$ are crystal structures of HMBS from other species and organisms. PDB entries 3ecr and 3eq1 are of human HMBS and are especially interesting because the mutations responsible for the geneticbased disease acute intermittent porphyria could be directly visualized in these human HMBS crystal structures. PDB entry $5 h 6 o$ is listed as 'to be published' and the PDB deposition is entitled Porphobilinogen deaminase from Vibrio cholerae.

$P D B$ entries 5ov4, 5ov5 and 5ov6 are crystal structures that each involve a mutant of the catalytic aspartic acid (Asp82 in Bacillus megaterium numbering). Guo et al. (2017) reported that 'the only mutant, D82E, which has the whole cofactor bound in a well ordered manner is catalytically active, while the other two (D82A and D82N) are not'.

Since 2018, several groups have reported studies of $\mathrm{ES}_{2}$ intermediate structures. These structures are as follows.

PDB entry 5m6r (Pluta et al., 2018) is a structure entitled Human porphobilinogen deaminase in complex with reaction

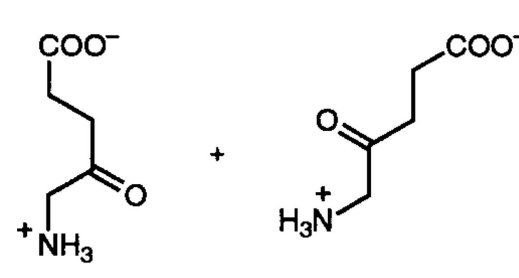

5-Aminolevulinic acid

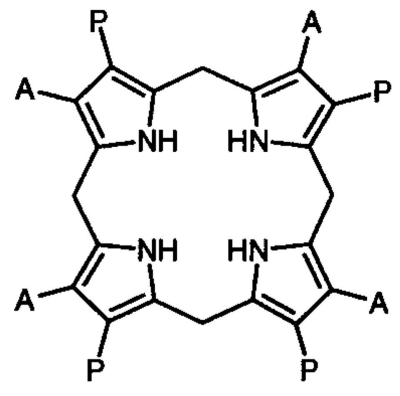

Uroporphyrinogen III
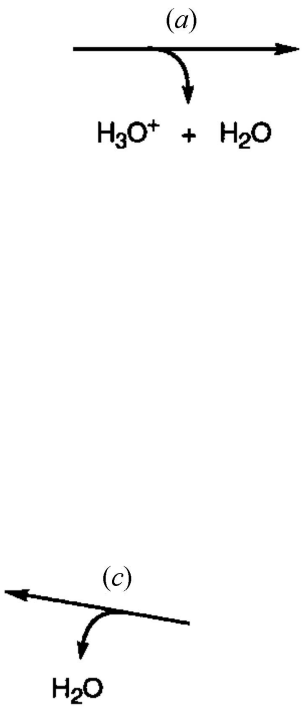

Figure 1

The biosynthesis of uroporphyrinogen III from 5-aminolevulinic acid. The enzymes involved are $(a)$ 5-aminolaevulinic acid dehydratase, (b) hydroxymethylbilane synthase (HMBS) and (c) uroporphyrinogen III synthase. A $=\mathrm{CH}_{2} \mathrm{COO}^{-} ; \mathrm{P}=\mathrm{CH}_{2} \mathrm{CH}_{2} \mathrm{COO}^{-}$. Reproduced from Hädener et al. (1999). 
intermediate $E S_{2}$. Note the $3.5 \AA$ change in the $c$ unit-cell parameter compared with PDB entry 5m7f, the holo enzyme.

PDB entry 7aak (Bustad et al., 2021) is a structure entitled Human porphobilinogen deaminase R173W mutant crystallized in the $\mathrm{ES}_{2}$ intermediate state. The maximum differences in unit-cell parameters compared with PDB entry 7aaj, the holo enzyme, are the values of $b$ and $c$, which differ by $1.5 \AA$, i.e. the $\mathrm{ES}_{2}$ intermediate was captured with only a small unit-cell change.

PDB entry 7cdo (Sato et al., 2021) is a structure entitled Crystal structure of the 2-iodoporphobilinogen-bound $E S_{2}$ intermediate form of human hydroxymethylbilane synthase. The crystals used by Sato and coworkers are shown in their Supplementary Fig. S3. 2-Iodoporphobilinogen (2-I-PBG) is described by Sato et al. (2021) as a noncompetitive inhibitor. From the same group of studies is PDB entry $7 \mathrm{ccy}$ entitled Crystal structure of the 2-iodoporphobilinogen-bound holo form of human hydroxymethylbilane synthase. Note the $7.4 \AA$

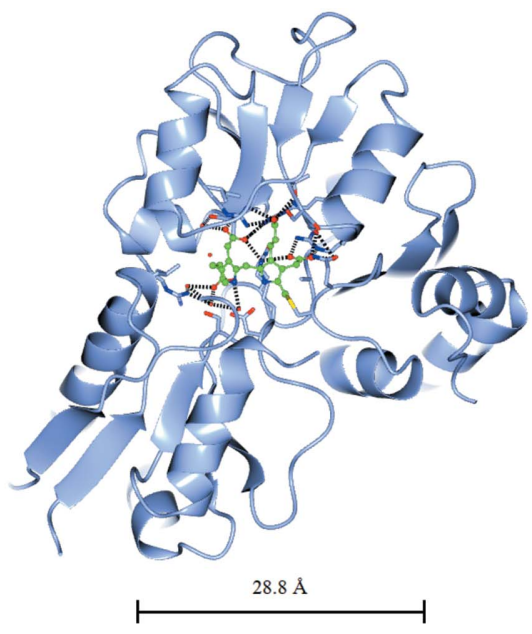

(a)
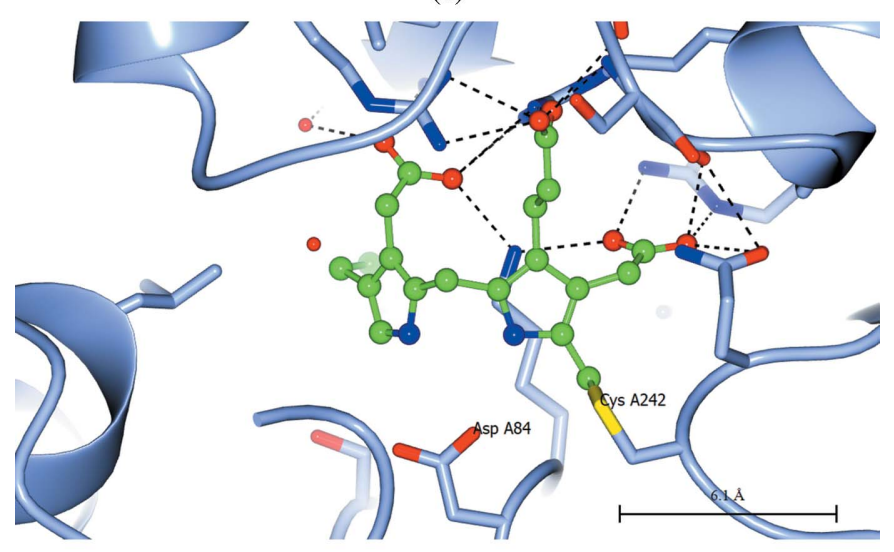

(b)

Figure 2

(a) The crystal structure of the active form of the E. coli HMBS enzyme in ribbon format (PDB entry 1ah5); the cofactor is in the middle of the picture. (b) An enlargement of the dipyrromethane cofactor; the righthand cofactor ring (referred to in the text as $\mathrm{C} 1$ ) is covalently attached to the labelled Cys242 (Cys261 in the human enzyme). The Asp side chain (Asp84 in E. coli HMBS and Asp99 in human HMBS) is visible just below the cofactor towards its left-hand side. This figure was produced by CCP4mg (McNicholas et al., 2011). difference in the $a$ unit-cell parameter compared with PDB entry $7 \mathrm{~cd} 0$. PDB entry $7 \mathrm{ccz}$ is entitled Crystal structure of the $E S_{2}$ intermediate form of human hydroxymethylbilane synthase and PDB entry 7ccx is entitled Crystal structure of the holo form of human hydroxymethylbilane synthase. Note that the $a$ unit-cell parameter of PDB entry $7 \mathrm{ccx}$ differs by $4 \AA$ compared with that of PDB entry 7ccy and by $11 \AA$ compared with those of PDB entries $7 \mathrm{ccz}$ and $7 \mathrm{cdo}$. In effect there are two unit-cell clusters here, albeit broadly similar. The use of 2-I-PBG by Sato et al. (2021) is interesting. They showed that it inhibited the HMBS reaction in a noncompetitive manner. Sato and coworkers reported that 'this contrasted with reported competitive and mixed-type inhibitors, such as 2-bromo-PBG and 6-methyl-PBG, respectively, which form covalent bonds with the cofactor and oligopyrrole chain, while 2-I-PBG does not form such'. They also reported that 'The overall structure of the 2-I-PBG-bound holo-HMBS was found to be similar to that of the inhibitor-free holo-HMBS'. Table 2 of Sato et al. (2021) provides comprehensive details of the interactions between pyrroles and the protein moiety in HMBS (in their four crystal structures). Fig. 8 of Sato et al. (2021) shows a predicted $\mathrm{ES}_{1}$ based on their crystal structure of human HMBS complexed with 2-I-PBG. As a historical note, bromoporphobilinogen was important in the study of the enzyme, as experiments by Warren \& Jordan (1988) using bromoporphobilinogen provided conclusive evidence for the direct covalent interaction of the substrate with the dipyrromethane (DPM) cofactor.

In mid-2021 the AlphaFold Protein Structure Database (AlphaFold DB; Jumper et al., 2021) was announced by DeepMind and the EMBL-EBI (the European Bioinformatics Institute, part of the European Molecular Biology Laboratory; https://alphafold.ebi.ac.uk/). This database spans 20 species and includes, for example, the predicted 3D structure of E. coli HMBS (Jumper et al., 2021; Tunyasuvunakool et al., 2021$)$. This includes a predicted structure for loop $45-57$, which has often been missing in the experimental structures.

\section{Discussion}

There are two principal themes in structural studies of this enzyme. The first is to unravel the details of the structural rearrangements during catalysis involving tetrapyrrole polymerization and release of a product (see Fig. 1) of precisely four units. The second theme is to relate the key amino-acid changes in the HMBS structure to the medical pathology acute intermittent porphyria (AIP). These two themes interrelate as the latter can be harnessed to inform the former, i.e. so as to trap intermediate states. Song et al. (2009) mapped a number of mutations that had been documented in the porphobilinogen deaminase (PBGD) gene of patients suffering from AIP onto their crystal structure of human PBGD (PDB entry 3ecr).

The method of mutagenesis to fully trap an enzyme intermediate state such as $\mathrm{ES}_{2}$ has the danger that the enzyme is effectively blocked and cannot move towards extending to the next step and on towards release of the product. The danger then is that the crystal structures are artefactual or, if not 


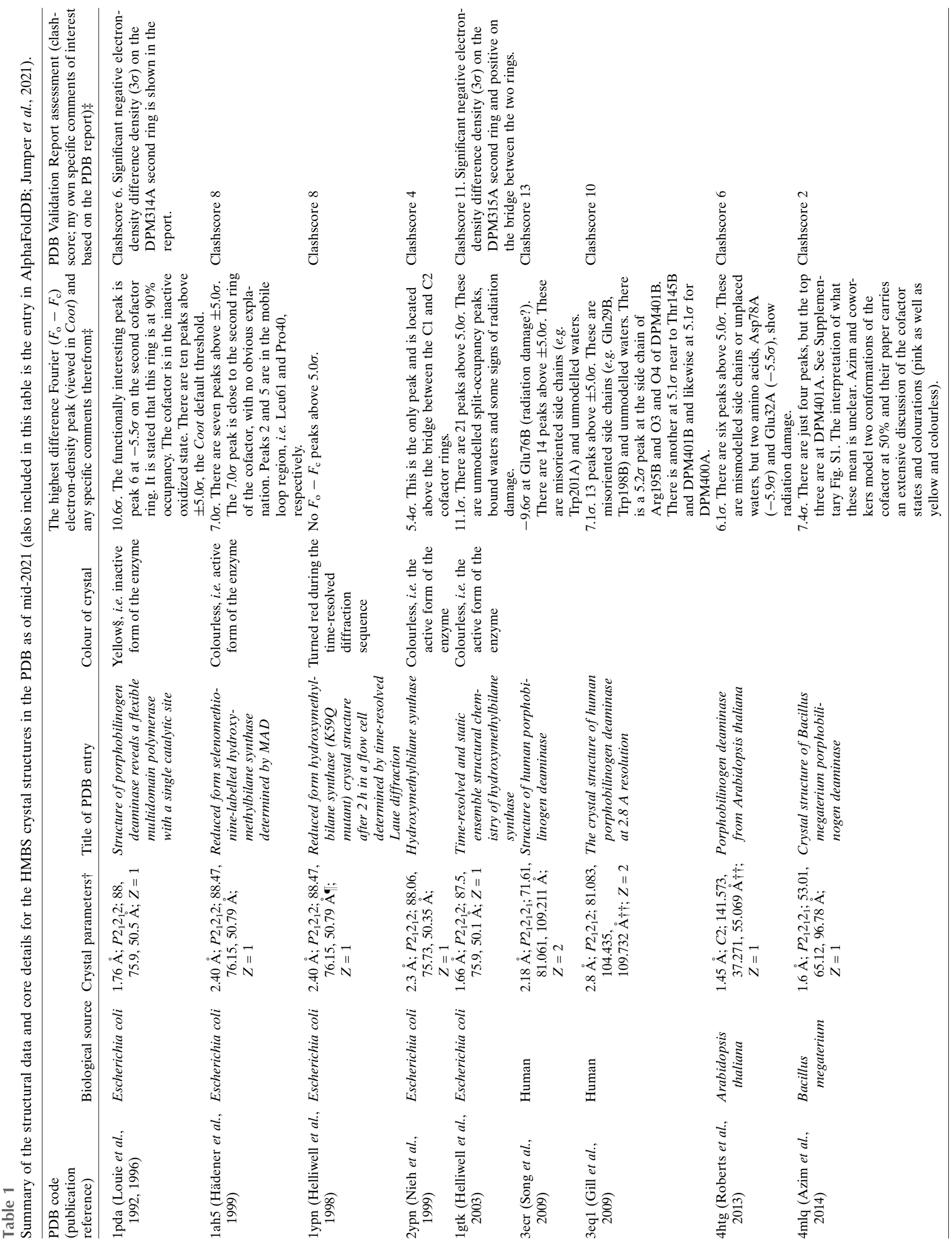



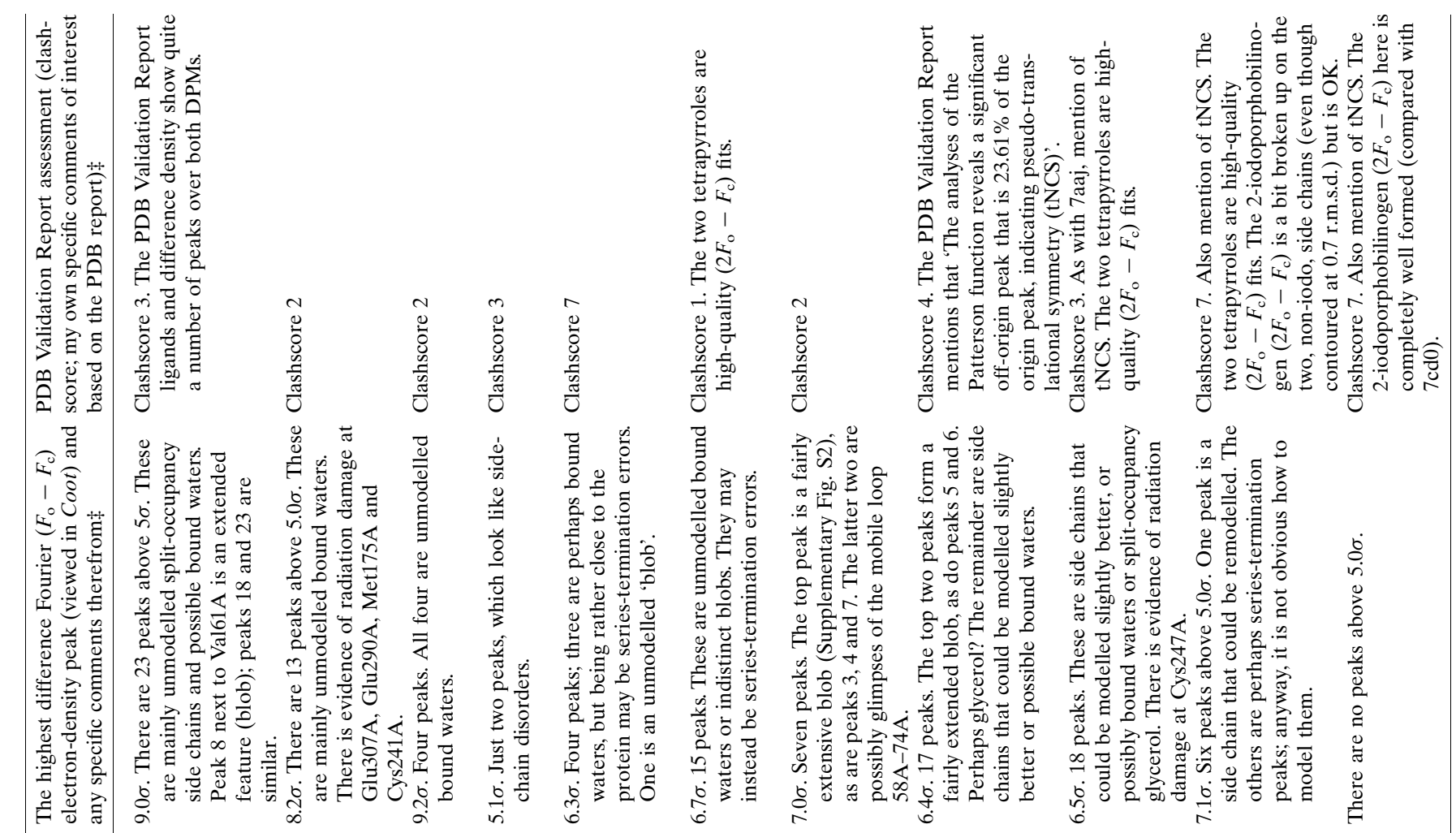

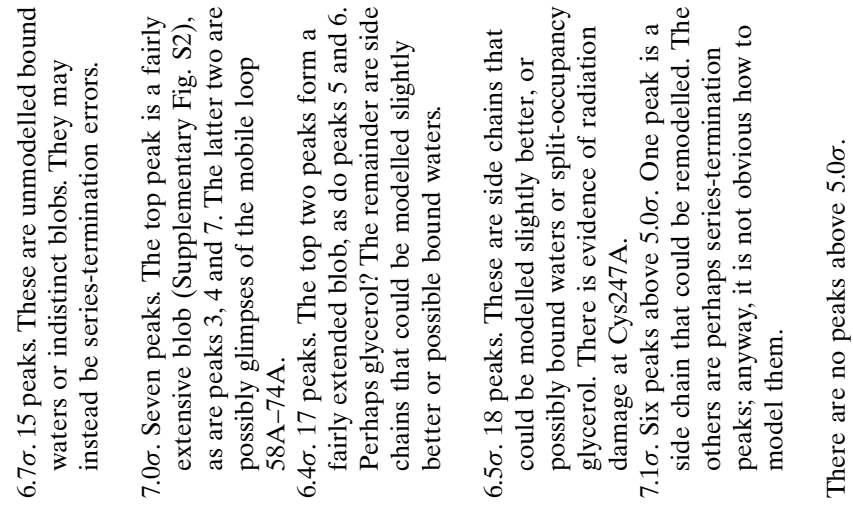
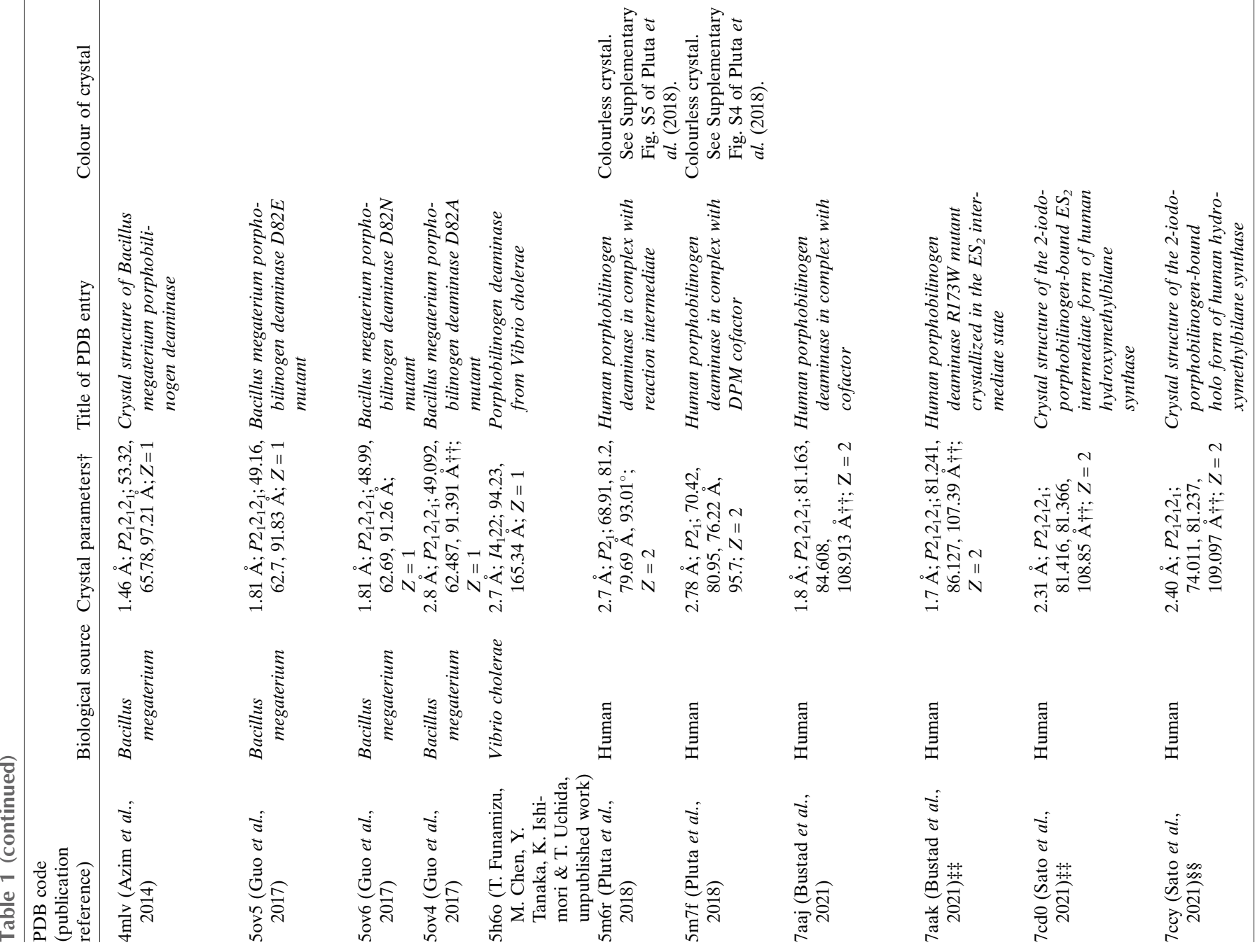


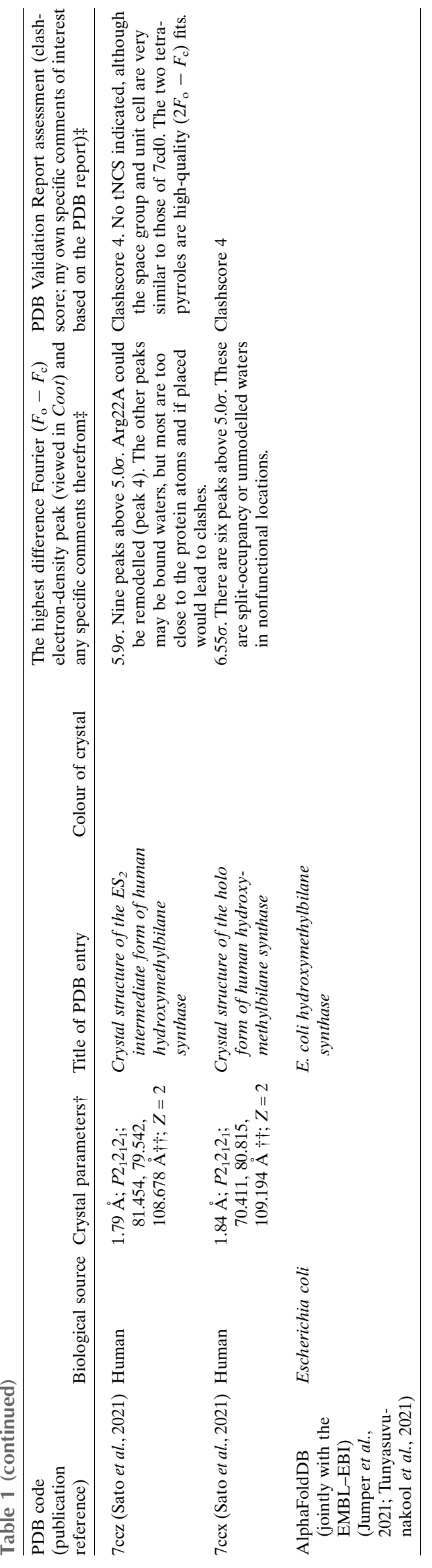

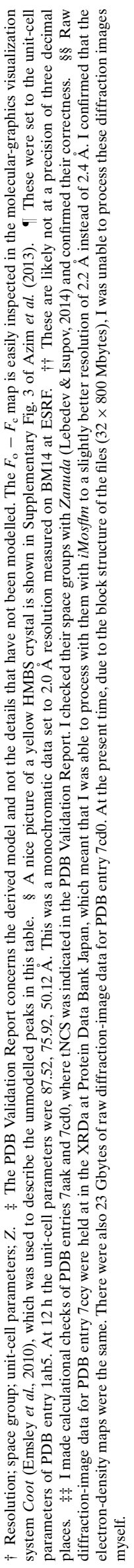

exactly that, then at least not natural. A mutant such as K59Q (Niemann et al., 1994) does not fully stop the enzyme at this second stage; rather, it slows the process down, which I think is a better approach to the natural state of the enzyme. In this case the X-ray diffraction experiment has to be sufficiently quick in its measurements to capture this accumulating population (Helliwell et al., 1998).

An alternative to time-resolved diffraction is to freeze-trap at the key stage of a sufficient accumulated intermediate, using the time sequence established by the flow-cell method. Alternatively, since the pre-formed crystal itself could restrict movements due to crystal packing, the consistency of the intermediate trapped structures could be explored in experiments repeated on different crystal forms.

Pluta et al. (2018) elegantly combine techniques to obtain results in solution using NMR for structural details and solution scattering for overall HMBS shape monitoring. These results 'suggest a reaction mechanism with localized segmental dynamics, ruling out the domain-reorienting mechanism'. Their crystallization was directly of the $\mathrm{ES}_{2}$ enzyme substrate intermediate eluted from a chromatography column (see Fig. 1 of Pluta et al., 2018). They explain that

\begin{abstract}
These results (crystal structures PDB 5m7f and 5m6r based on 3ecr of Song et al., 2009), combined with the SAXS and NMR experiments, allow us to propose a mechanism for the reaction progression that requires less structural rearrangements than previously suggested [by Louie et al. (1992)]: the enzyme slides a flexible loop (R255-V263) over the growing-product active site cavity ... The large number of AIP-causing mutations occurring in this loop confirms the key relevance of this structural element not only for the reaction but also in disease.
\end{abstract}

For comparison purposes with E. coli, the sequence of this key loop Arg255-Val263 in the human enzyme would be Asn235Gln243, encompassing Cys242, which is covalently attached to the cofactor ring C1 (see PDB entries 1ah5, 1ypn and 2ypn). It is this covalently linked cysteine (Cys261) that pulls the cofactor past the catalytic Asp99 (Asp84 in E. coli). Supplementary Figs. S4 and S5 of Pluta et al. (2018) show the crystals of $\mathrm{E}_{\text {holo }}$ and $\mathrm{ES}_{2}$, which are colourless.

\section{Consistencies and inconsistencies in the crystal structures}

In addressing the goal of elucidating the mechanism of the enzyme, we can ask: what are the consistencies and inconsistencies in the crystal structures thus far? Here we are mainly addressing the group of crystal structures with PDB codes 1ypn, 5m6r, 7aaj, 7aak, 7cdo and 7ccz.

\subsection{Consistency of the active-site structures of the $\mathrm{ES}_{2}$ intermediate}

Firstly, Fig. 3 shows the best least-squares-calculated overlay of molecule $A$ of the cofactor plus $\mathrm{ES}_{2}$ in PDB entries $5 \mathrm{~m} 6 \mathrm{r}$ and $7 \mathrm{aak}$. These structures, which are both of human HMBS, are remarkably consistent considering that they were obtained by two different groups in two different space groups 
and one is an Arg173Trp mutant form of the enzyme. This mutation was chosen as it prevents the formation of $\mathrm{ES}_{3}$ [see Fig. 2(d) of Bustad et al. (2021), which is a mass spectrum showing only $\mathrm{ES}_{2}$ ]. These two crystal structures agree that the new pyrrole rings $\mathrm{S} 1$ and $\mathrm{S} 2$ occupy the original positions of the $\mathrm{C} 1$ and $\mathrm{C} 2$ rings of the DPM cofactor in the HMBS enzyme structure.

An important observation of Bustad et al. (2021) is in the caption to their Supplementary Fig. S5, where they state that 'Upon the movement of cofactor-binding loop from $\mathrm{E}_{\text {holo }}$ to

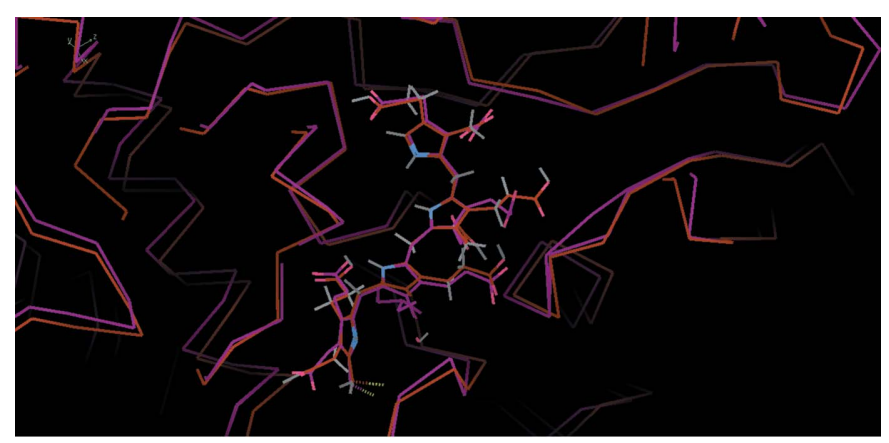

(a)

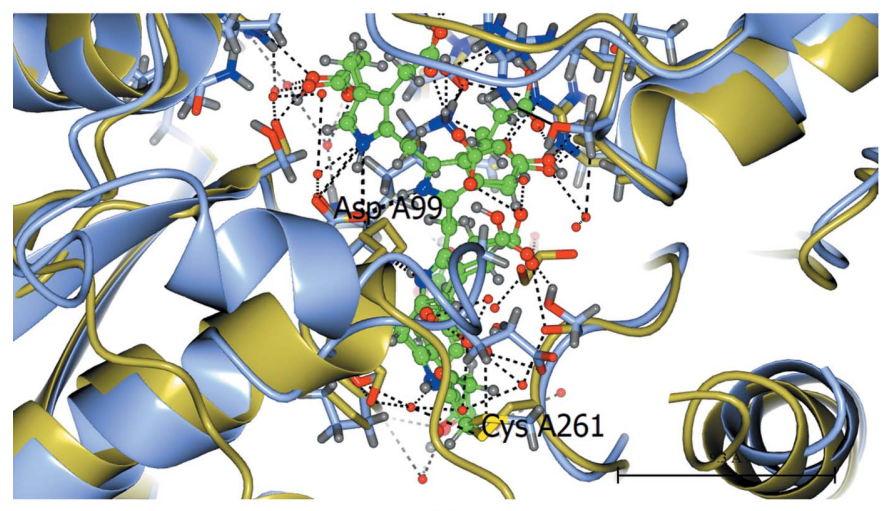

(b)

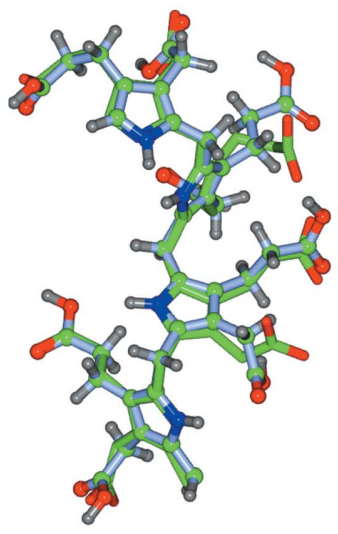

(c)

Figure 3

Best least-squares-calculated overlay of the cofactor plus $\mathrm{ES}_{2}$ for human HMBS, PDB entries 5m6r and human 7aak, in molecule $A$. Molecule $B$ also shows a similarly good agreement between PDB entries $5 \mathrm{~m} 6 \mathrm{r}$ and 7aak. (a) was made with Coot (Emsley et al., 2010), and (b) and (c) were made with CCP4mg (McNicholas et al., 2011). All three show very similar orientations and show complementary information. Cys 261 is covalently linked to the first ring of the cofactor. The least-squares-calculated overlay of PDB entries 5m6r and 7aak used the five amino acids centred on Asp99.
$\mathrm{ES}_{2}$ only small change in Val263 can be detected'. This is shown in Fig. 4, which emphasizes that it is the motion of Cys261 itself that is largely responsible for pulling the cofactor to make room for the addition of two PBG molecules to form $\mathrm{ES}_{2}$.

Fig. 5 documents similar agreement for the cofactor plus $\mathrm{ES}_{2}$ for PDB entries 5m6r and 7cd0. Similar agreement for $\mathrm{ES}_{2}$ is shown by PDB entry $7 \mathrm{ccz}$.

\subsection{Time-resolved diffraction experiment on the Lys59GIn mutant of HMBS to accumulate $\mathrm{ES}_{2}$}

PDB entry 1ypn from the time-resolved study showed the growth of an extended electron density from the region of Asp84 and cofactor ring C2. Like those used for PDB entry 1ah5, the crystals used for PDB entry 2ypn were colourless before the flow of PBG over the crystals, with the cofactor in the active state. In the case of PDB entry 1ypn, after $2 \mathrm{~h}$ of PBG flow the crystals became pink/red (see Section 4.2.4 of Helliwell et al., 1998). To achieve the full red colour at $12 \mathrm{~h}$ requires release of the product and cyclization of the tetrapyrrole. After the PBG supply had been turned off at the $2 \mathrm{~h}$ time point, a crystal structure was determined at $12 \mathrm{~h}$. The extended electron density that grew in the active site by $2 \mathrm{~h}$ had disappeared, whilst the crystal at $12 \mathrm{~h}$ was red. How do we reconcile these observations with the agreed model (Figs. 3, 5 and 6) of the cysteine bond (Cys261 in human HMBS, corresponding to Cys 242 in E. coli $\mathrm{HMBS}$ ) to cofactor ring C1

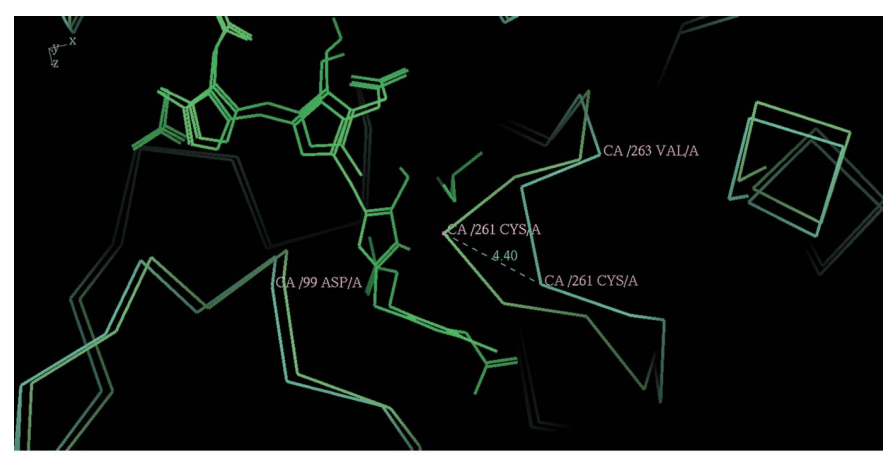

(a)

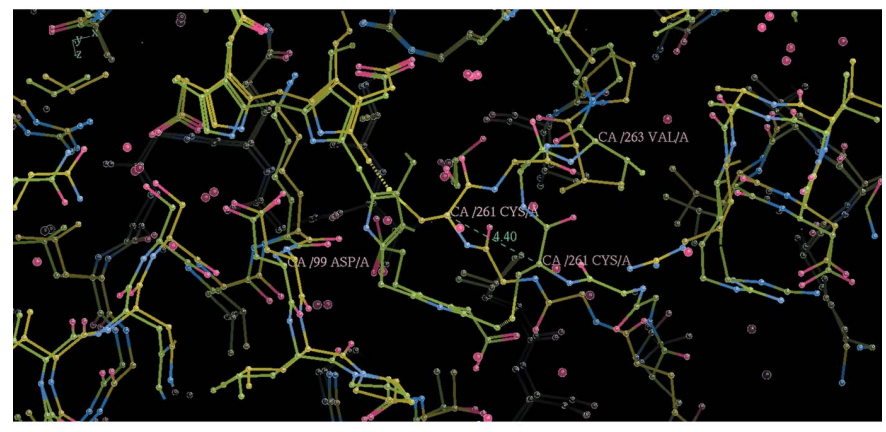

Figure 4

(b)

The motion of (human) Cys261 itself is largely responsible for pulling the cofactor to make room for the addition of two PBG molecules to form $\mathrm{ES}_{2}$ (Bustad et al., 2021). The movement of Cys261 (PDB entries 7aaj and 7 aak, molecules $A$ ) is $4.4 \AA$ and that of Val263 is $0.8 \AA$. The movement of Asp99 is $0.5 \AA$. (a) shows the alpha carbons and the cofactors and $\mathrm{ES}_{2} ;(b)$ shows an identical view with all atoms. 
pulling the growing pyrrole chain so that S1 and S2 occupy the sites originally occupied by $\mathrm{C} 1$ and $\mathrm{C} 2$ ? One option referred to in Helliwell et al. (1998) was that the red colour arises from the enzyme molecules on the crystal surface only and therefore the extended electron density that grew in the active site was only the arrival of PBG in the active site. Indeed, there was no bond to the $\mathrm{C} 2$ ring, which would support this; i.e. it was a prereactive species. The results shown in Figs. 3, 5 and 6 suggest a new possibility: that the reaction in the crystal from which PDB entry 1ypn was obtained had gone to EP, the cofactor had returned to its starting position, and the bond between S4 and the cofactor $\mathrm{C} 2$ ring would also have been broken. This is only viable if Cys 242 and the nearby loop residues 243 onwards were able to move unrestrictedly, i.e. if there was a solvent channel directly above the loop. Otherwise, the loop would fight against the crystal packing and the crystal would most likely have broken up. Fig. 6 shows that there is a solvent channel directly above this loop. Against this second hypothesis is that we did not directly see sliding of the DPM cofactor or of the Cys 242 associated with it (at most $0.1 \AA$ at 8 min; see Fig. 4 of Helliwell et al., 1998).

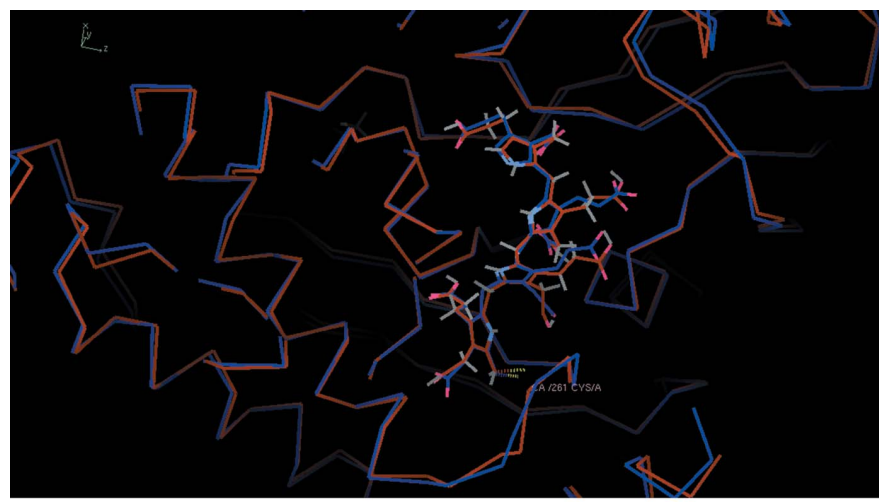

(a)

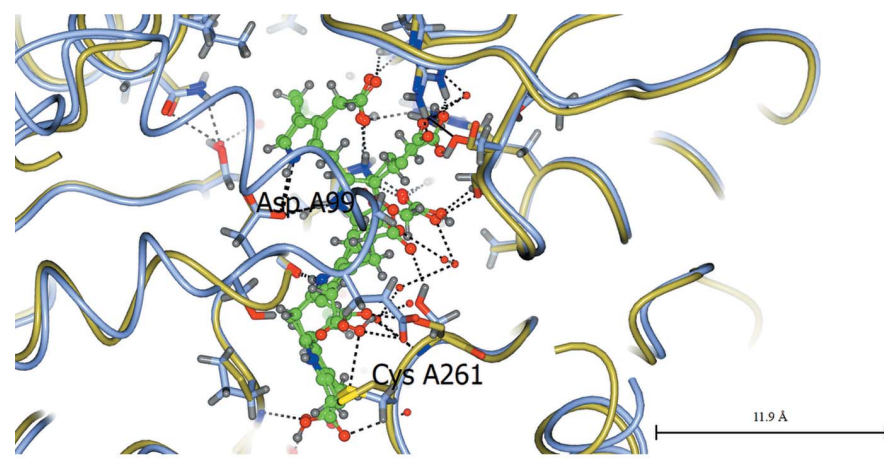

(b)

Figure 5

Best least-squares-calculated overlay of the cofactor plus $\mathrm{ES}_{2}$ for human HMBS, PDB entries 5m6r and human 7cd0, in molecule $A$. Molecule $B$ shows very similar agreement. Cys 261 is at the lower middle and thereby also identifies the first ring of the cofactor. Note that the iodinated PBG inhibitor is in molecule B. (a) was made with Coot (Emsley et al., 2010) and (b) was made with CCP4mg (McNicholas et al., 2011). Both show very similar orientations and show complementary information. Cys 261 is covalently linked to the first ring of the cofactor. The least-squarescalculated overlay of PDB entries $5 \mathrm{~m} 6 \mathrm{r}$ and $7 \mathrm{~cd} 0$ used the five amino acids centred on Asp99.
To return to the visual observations made in Section 4.2 .4 of Helliwell et al. (1998): 'In separate experiments involving the soaking of a wild-type HMBS crystal in a pot of solution the pink/red colour developed in similar fashion to the flow cell experiments.' Furthermore,

After $c a .24 \mathrm{~h}$ the crystal showed distinct cracks and the surrounding solution also became gradually pink within 13-24 h, but markedly more slowly than the crystal (results of A. Hädener). These observations are consistent with formation of a cyclized oxidized product in the crystal, which may or may not be released from the active site. It cannot be ruled out, however, that non-enzymatic formation of tetrapyrrole in solution and subsequent cyclization and oxidation processes are followed by the absorption of the oxidized material by the crystal, thus concentrating it like a red dye.

It is also interesting that Azim et al. (2014) contains an extensive discussion of their cofactor states and colourations (pink as well as yellow). They noted an intriguing pink colouration of the freshly purified protein which gradually changes to yellow over a 2-3-week period.'

\subsection{What is the role of the loop 61-76 (human HMBS numbering)?}

PDB entries 4htg, 5m6r and 7aak are exceptions in that they show the ordering of this loop. Actually, however, PDB entries 5m6r and 7aak, which each have two HMBS molecules in their asymmetric units, have disordered loops in one of these two HMBS molecules. Yet both PDB entries 5m6r and 7aak have their two HMBS molecules with fully formed $\mathrm{C} 1+\mathrm{C} 2+\mathrm{S} 1+\mathrm{S} 2$ in their $\mathrm{ES}_{2}$ crystal structures. This suggests that the role of this loop is incidental to the catalytic sequence of events, at least up to $\mathrm{ES}_{2}$. However, Sato et al. (2021) report that 'flexibility of this loop in the proximity of the active site appears to be involved in the binding of 2-I-PBG and the substrate, although no direct interactions between the loop (residues 5869) and 2-I-PBG were observed'. This last sentence seems to be a little self-contradictory.

\subsection{What can be said about the $\mathrm{ES}_{2}$ to $\mathrm{ES}_{3}$ step?}

As the experimental design in Bustad et al. (2021) involved the R173W mutant and they showed conclusively that $\mathrm{ES}_{3}$ was

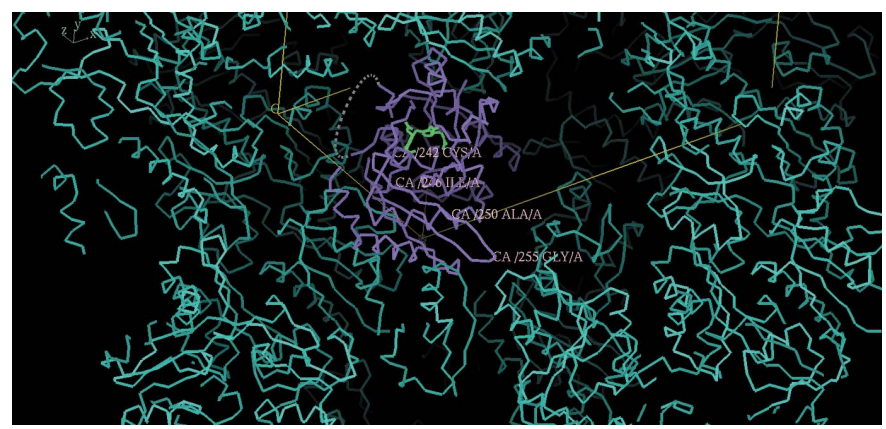

Figure 6

Crystal-packing diagram for E. coli (PDB entry 1ypn) showing the solvent channel directly above the 242-255 polypeptide loop of HMBS. Note that the lattice neighbour of Gly255 is Gly33 and residue 32 is a proline, i.e. it is unlikely to interfere with loop movement. 
not produced, then it is compelling to agree with Bustad et al. (2021) that 'the substrate elongation from $\mathrm{ES}_{2}$ to $\mathrm{ES}_{3}$ is crucially dependent on Arg173'. Secondly, since Pluta et al. (2018) actually crystallized $\mathrm{ES}_{2}$ for the wild-type enzyme and Fig. 3 shows the agreement between the two approaches, this lends further support to the crucial role of Arg173.

Sato et al. (2021) came the closest to a crystal structure of $\mathrm{ES}_{3}$; they reported that they 'attempted crystallization and structure analysis of $\mathrm{ES}_{3}$ intermediate of HMBS, and successfully obtained its crystals. However, structural analysis of the $\mathrm{ES}_{3}$ intermediate has not yet been successful due to its instability.' However, they make the very plausible conclusion from their several crystal structures that 'Since 2-I-PBG is present at the same site in both structures (holo hHMBS and $\mathrm{ES}_{2}$ ), it is considered that each of the four substrate molecules binds to a single substrate-binding site in HMBS and is condensed consecutively on the DPM cofactor in four successive reactions.' This assertion then provides a definite suggestion regarding $\mathrm{ES}_{3}$ and $\mathrm{ES}_{4}$ formation. Also, it should be considered that the step from $\mathrm{ES}_{2}$ to $\mathrm{ES}_{3}$ requires further rearrangement, which cannot happen in the crystal state. Therefore, $\mathrm{ES}_{3}$, stabilized somehow, may need to be formed before crystallization.

\subsection{What can be said about how the product is released?}

Bung et al. (2014, 2018, 2019) undertook moleculardynamics (MD) studies. Fig. 7 from Bung et al. (2018) and the associated text describe three possible routes for the formed product to exit the enzyme. Specifically, they state that ' $R 167$ acts as a gatekeeper for the HMB exit'. In his PhD thesis, Nieh offered a similar assertion based on the $2 \mathrm{~h}$ time-resolved experiment (Nieh, 1997):

The elongated peak passes the critical residues Arg149, which is important in forming $\mathrm{ES}_{2}$, and approaches $\operatorname{Arg} 155$, which is important in the $\mathrm{ES}_{4}$ to EP step, and then projects out into the solvent channel. Residues Arg149 and Arg155 in E. coli are equivalent to residues 167 and 173 in the human HMBS enzyme and mutation of these two residues can cause acute intermittent porphyria. The modelling of EP is shown in Fig. 6.13 [reproduced here as Fig. 7] with the final omit map overlaid. Note that only ring A gives a reasonably detailed fit.

\subsection{Consistency given by combining different methods}

Bustad et al. (2021) elegantly show how high-resolution mass spectrometry allows direct analysis of the intermediates in order to directly plan the X-ray crystal structures. A weakness of an X-ray crystal structure is that where there is disorder, such as a mobile loop or a floppy tripyrrole or tetrapyrrole, there will be broken up or missing electron density. However, if the mass is directly measured then this datum assumes a special importance. This approach of measuring the mass was also performed by Sato et al. (2021).

The role of SAXS where there might be large-scale interdomain movements, as originally proposed for HMBS by Louie et al. (1992), can be definitive and shows what is happening in solution. However, if the mechanism has been shown to be the localized movement of Cys261, without interdomain movements, then SAXS will be insensitive to such localized changes.

The role of monitoring the colour, from colourless to pink to full red, is illustrated well in the efforts to unravel the mechanism of HMBS. The colour changes have been emphasized several times above.

X-ray crystal structures at these diffraction resolutions cannot show the $\mathrm{H}$ atoms of ionizable groups, or even at ultrahigh resolution if the side chains are a little mobile. [Nonionizable groups can have their $\mathrm{H}$ atoms placed in calculated positions with confidence.] Since molecular-dynamics
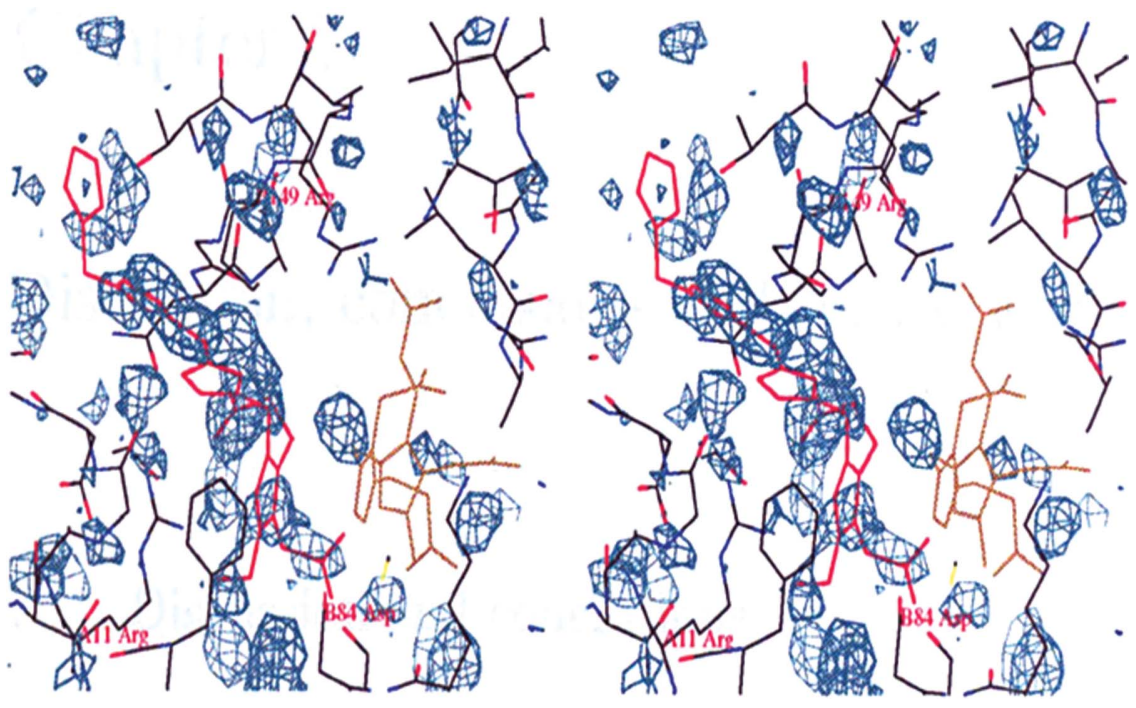

Figure 7

Stereoview of the modelling of the Michaelis complex EP (red) overlaid with the $2 \mathrm{~h}$ time point $\left(F_{\mathrm{o}}-F_{\mathrm{c}}\right)$ electron-density omit map contoured at $2.00 \sigma$. From Nieh (1997). The acetate and propionate side chains of the second, third and fourth pyrroles could not be placed due to insufficient detail in the density map. 
simulations propose a mechanism relying on the protonation of incoming PBG molecules by Arg26, and electrophilic addition and deprotonation in concert with Asp99 (Bung et al., 2018, 2019), then experiments seeking to place these $\mathrm{H}$ atoms, and as a function of time, are the most challenging of all. Neutron crystallography of suitably trapped intermediate states through wet chemistry or freeze-trapping has not yet been applied. These experiments would require larger crystals to be grown than hitherto. It is also worth noting that the $\mathrm{pH}$ optimum of the E. coli HMBS enzyme is 7.4-8.0, yet the best crystallization conditions thus far were at $\mathrm{pH}$ 5.3. As Hädener et al. (1999) remark

The pH optimum for HMBS from E. coli is between 7.4 and 8.0, and the isoelectric point is 4.5 . Within the optimal $\mathrm{pH}$ interval and for the overall reaction, the Michaelis constant is between 5 and $20 \mathrm{mM}$, and the turnover constant is of the order of $0.1 \mathrm{~s}^{-1}$ with respect to the formation of HMB.

Functionally relevant protonation states will require crystals that are grown under functionally appropriate conditions.

To summarize, the human HMBS crystallization conditions, and in one case the solution for SAXS measurements, were as follows.

Song et al. (2009) state in PDB entry 3ecr that the pH of their human HMBS crystallization was 8.0 (this was not stated in their paper).

The crystallization conditions used by Pluta et al. (2018) for human HMBS were between $\mathrm{pH} 6.5$ and 7.2, and their SAXS solution was at $\mathrm{pH} 8.5$.

The crystallization conditions used by Bustad et al. (2021) for human HMBS were at $\mathrm{pH}$ 5.1.

The crystallization conditions used by Sato et al. (2021) for human HMBS were at $\mathrm{pH}$ 8.3.

In addition to the extensive MD simulations of Bung et al. (2019) described above, MD calculations of the $\mathrm{ES}_{2}$ intermediate were also undertaken by Sato et al. (2021). These suggested that thermal fluctuation of the lid (residues 58-75, human numbering) and cofactor-binding loops causes the substrate recruitment and oligopyrrole chain shift needed for consecutive condensation. This suggestion relates to the observation by Bustad et al. (2021) that Cys261 is the locus of the movement of the cofactor during catalysis, but what is the 'engine' or energy to drive this movement? Perhaps Sato et al. (2021) have the answer, namely thermal fluctuation (of the lid and cofactor-binding loops). However, thermal fluctuations are random displacements rather than being purposeful in a particular direction. The MD simulations performed by Sato et al. (2021) required the protonation states of Glu223 and His160; at these diffraction resolutions these needed to be predicted, which was performed using the $H++$ server (http:// biophysics.cs.vt.edu). An evaluation of the predictive accuracy of several prediction servers has been undertaken by Fisher $e t$ al. (2009), who concluded that predicting histidine protonation was especially difficult. Sato et al. (2021) provide three movie files; the first two show the jittery nature of a wide suite of atomic motions and the third movie, of the $\mathrm{ES}_{2}$ in HMBS in skeletal format, very clearly shows the simulated dynamic role of loops.

\section{Future directions}

Experimental capabilities for time-resolved diffraction have expanded considerably. The new extremely bright synchrotronradiation sources such as those at MAX IV and ESRF will allow smaller protein crystal sizes. In the experiments of Helliwell et al. (1998) the E. coli HMBS crystals were typically $0.5 \times 0.5 \times 0.05 \mathrm{~mm}$ in size. The human HMBS crystals used by Sato et al. (2021) were $\sim 0.2 \times 0.2 \times 0.02 \mathrm{~mm}$ for the inhibitor-free holo form and $\sim 0.1 \times 0.1 \times 0.01 \mathrm{~mm}$ for the inhibitor-free $\mathrm{ES}_{2}$ intermediate. The diffusion time of $\mathrm{PBG}$ into the crystal for smaller crystals would be speeded up accordingly. These would also likely lead to better quality (less mosaic) freeze-trapping of crystals in intermediate states. This said, there is a growing awareness of examples of cryoartefacts which must be guarded against, such as incorrectly placed side chains and their bound waters (see, for example, Halle, 2004).

The availability of high-quality protein fold and structure predictions across multiple species from AlphaFold DB (Jumper et al., 2021; Tunyasuvunakool et al., 2021) will allow further insights into the mobile loops in HMBS that have challenged experimental methods. Secondly, these predicted structures may well allow new crystallization constructs that can be used to explore different crystal packings and further crystallizations at the functional $\mathrm{pH}$ of the enzyme. Such crystallization constructs would allow new avenues to be explored to grow larger crystals for neutron crystallography, as well as the application of macroseeding methods (Chayen et al., 2010).

The community of researchers interested in this fascinating enzyme is growing, as shown by the pace of entries in Table 1 . I hope that this topical review proves useful to further expand this community.

\section{Acknowledgements}

I am indebted to Dr Alfons Hädener, formerly of the University of Basle Chemistry Department, for his great collaborator support and subsequent regular discussions over many years. My research in this area had various funding support. So, I gratefully acknowledge the Science and Engineering Research Council and the Engineering and Physical Sciences Research Council, the Biology and Biotechnology Sciences Research Council, The Wellcome Trust and the EU for research grants. I also thank the British Government for the provision of an award to Y.-P. Nieh, and the support of his family. Dr A. Hädener, with whom I collaborated, gratefully acknowledges the financial support from the Swiss National Science Foundation and the Ciba Geigy-Jubiläums-Stiftung, and the Stipendienfonds der Basler Chemischen Industrie. We thank the SRS Daresbury Laboratory and the ESRF for synchrotron radiation beamtime and the support laboratory of the EMBL Grenoble. 


\section{References}

Azim, N., Deery, E., Warren, M. J., Erskine, P., Cooper, J. B., Wood, S. P. \& Akhtar, M. (2013). Acta Cryst. F69, 906-908.

Azim, N., Deery, E., Warren, M. J., Wolfenden, B. A. A., Erskine, P., Cooper, J. B., Coker, A., Wood, S. P. \& Akhtar, M. (2014). Acta Cryst. D70, 744-751.

Bung, N., Pradhan, M., Srinivasan, H. \& Bulusu, G. (2014). PLoS Comput. Biol. 10, e1003484.

Bung, N., Roy, A., Chen, B., Das, D., Pradhan, M., Yasuda, M., New, M. I., Desnick, R. J. \& Bulusu, G. (2018). Proc. Natl Acad. Sci. USA, 115, E4071-E4080.

Bung, N., Roy, A., Priyakumar, U. D. \& Bulusu, G. (2019). Phys. Chem. Chem. Phys. 21, 7932-7940.

Bustad, H. J., Kallio, J. P., Laitaoja, M., Toska, K., Kursula, I., Martinez, A. \& Jänis, J. (2021). iScience, 24, 102152.

Cassetta, A., Deacon, A. M., Ealick, S. E., Helliwell, J. R. \& Thompson, A. W. (1999). J. Synchrotron Rad. 6, 822-833.

Chayen, N. E., Helliwell, J. R. \& Snell, E. H. (2010). Macromolecular Crystallization and Crystal Perfection. Oxford University Press.

Emsley, P., Lohkamp, B., Scott, W. G. \& Cowtan, K. (2010). Acta Cryst. D66, 486-501.

Fisher, S. J., Wilkinson, J., Henchman, R. H. \& Helliwell, J. R. (2009). Crystallogr. Rev. 15, 231-259.

Gill, R., Kolstoe, S. E., Mohammed, F., Al d-Bass, A., Mosely, J. E., Sarwar, M., Cooper, J. B., Wood, S. P. \& Shoolingin-Jordan, P. M. (2009). Biochem. J. 420, 17-25.

Guo, J., Erskine, P., Coker, A. R., Wood, S. P. \& Cooper, J. B. (2017). Acta Cryst. F73, 612-620.

Hädener, A., Matzinger, P. K., Battersby, A. R., McSweeney, S., Thompson, A. W., Hammersley, A. P., Harrop, S. J., Cassetta, A., Deacon, A., Hunter, W. N., Nieh, Y. P., Raftery, J., Hunter, N. \& Helliwell, J. R. (1999). Acta Cryst. D55, 631-643.

Hädener, A., Matzinger, P. K., Malashkevich, V. N., Louie, G. V., Wood, S. P., Oliver, P., Alefounder, P. R., Pitt, A. R., Abell, C. \& Battersby, A. R. (1993). Eur. J. Biochem. 211, 615-624.

Halle, B. (2004). Proc. Natl Acad. Sci. USA, 101, 4793-4798.

Helliwell, J. R., Nieh, Y.-P., Habash, J., Faulder, P. F., Raftery, J., Cianci, M., Wulff, M. \& Hädener, A. (2003). Faraday Discuss. 122, 131-144.

Helliwell, J. R., Nieh, Y.-P., Raftery, J., Cassetta, A., Habash, J., Carr, P. D., Ursby, T., Wulff, M., Thompson, A. W., Niemann, A. C. \& Hädener, A. (1998). Faraday Trans. 94, 2615-2622.

Jumper, J., Evans, R., Pritzel, A., Green, T., Figurnov, M., Ronneberger, O., Tunyasuvunakool, K., Bates, R., Žídek, A.,
Potapenko, A., Bridgland, A., Meyer, C., Kohl, S. A. A., Ballard, A. J., Cowie, A., Romera-Paredes, B., Nikolov, S., Jain, R., Adler, J., Back, T., Petersen, S., Reiman, D., Clancy, E., Zielinski, M., Steinegger, M., Pacholska, M., Berghammer, T., Bodenstein, S., Silver, D., Vinyals, O., Senior, A. W., Kavukcuoglu, K., Kohli, P. \& Hassabis, D. (2021). Nature, 596, 583-589.

Lebedev, A. A. \& Isupov, M. N. (2014). Acta Cryst. D70, 2430-2443.

Louie, G. V., Brownlie, P. D., Lambert, R., Cooper, J. B., Blundell, T. L., Wood, S. P., Warren, M. J., Woodcock, S. C. \& Jordan, P. M. (1992). Nature, 359, 33-39.

Louie, G. V., Brownlie, P. D., Lambert, R., Cooper, J. B., Blundell, T. L., Wood, S. P., Malashkevich, V. N., Hädener, A., Warren, M. J. \& Shoolingin-Jordan, P. M. (1996). Proteins, 25, 48-78.

McNicholas, S., Potterton, E., Wilson, K. S. \& Noble, M. E. M. (2011). Acta Cryst. D67, 386-394.

Nieh, Y.-P. (1997). PhD thesis. University of Manchester, UK. https:// ethos.bl.uk/OrderDetails.do?uin=uk.bl.ethos.680176.

Nieh, Y. P., Raftery, J., Weisgerber, S., Habash, J., Schotte, F., Ursby, T., Wulff, M., Hädener, A., Campbell, J. W., Hao, Q. \& Helliwell, J. R. (1999). J. Synchrotron Rad. 6, 995-1006.

Niemann, A. C., Hädener, A. \& Matzinger, P. K. (1994). Helv. Chim. Acta, 77, 1791-1809.

Pluta, P., Roversi, P., Bernardo-Seisdedos, G., Rojas, A. L., Cooper, J. B., Gu, S., Pickersgill, R. W. \& Millet, O. (2018). Biochim. Biophys. Acta, 1862, 1948-1955.

Roberts, A., Gill, R., Hussey, R. J., Mikolajek, H., Erskine, P. T., Cooper, J. B., Wood, S. P., Chrystal, E. J. T. \& Shoolingin-Jordan, P. M. (2013). Acta Cryst. D69, 471-485.

Sato, H., Sugishima, M., Tsukaguchi, M., Masuko, T., Iijima, M., Takano, M., Omata, Y., Hirabayashi, K., Wada, K., Hisaeda, Y. \& Yamamoto, K. (2021). Biochem. J. 478, 1023-1042.

Song, G., Li, Y., Cheng, C., Zhao, Y., Gao, A., Zhang, R., Joachimiak, A., Shaw, N. \& Liu, Z. J. (2009). FASEB J. 23, 396-404.

Tunyasuvunakool, K., Adler, J., Wu, Z., Green, T., Zielinski, M., Žídek, A., Bridgland, A., Cowie, A., Meyer, C., Laydon, A., Velankar, S., Kleywegt, G. J., Bateman, A., Evans, R., Pritzel, A., Figurnov, M., Ronneberger, O., Bates, R., Kohl, S. A. A., Potapenko, A., Ballard, A. J., Romera-Paredes, B., Nikolov, S., Jain, R., Clancy, E., Reiman, D., Petersen, S., Senior, A. W., Kavukcuoglu, K., Birney, E., Kohli, P., Jumper, J. \& Hassabis, D. (2021). Nature, 596, 590-596.

Warren, M. J. \& Jordan, P. M. (1988). Biochemistry, 27, 90209030 . 